\title{
Total Analysis of the Major Secoiridoids in Extra Virgin Olive Oil: Validation of an UHPLC-ESI-MS/MS Method
}

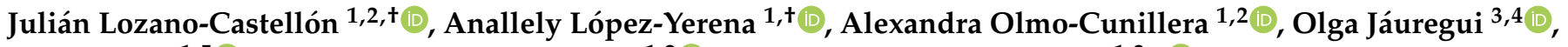 \\ Maria Pérez ${ }^{1,5}{ }^{(0)}$, Rosa $M^{a}$ Lamuela-Raventós ${ }^{1,2}\left(\mathbb{D}\right.$ and Anna Vallverdú-Queralt ${ }^{1,2, *(1)}$
}

Citation: Lozano-Castellón, J.; López-Yerena, A.; Olmo-Cunillera, A.; Jáuregui, O.; Pérez, M.;

Lamuela-Raventós, R.M.;

Vallverdú-Queralt, A. Total Analysis of the Major Secoiridoids in Extra Virgin Olive Oil: Validation of an UHPLC-ESI-MS/MS Method. Antioxidants 2021, 10, 540. https:// doi.org/10.3390/antiox10040540

Academic Editors: Vlasios Goulas and Alexandra Barbouti

Received: 16 March 2021

Accepted: 26 March 2021

Published: 30 March 2021

Publisher's Note: MDPI stays neutral with regard to jurisdictional claims in published maps and institutional affiliations.

Copyright: (c) 2021 by the authors. Licensee MDPI, Basel, Switzerland. This article is an open access article distributed under the terms and conditions of the Creative Commons Attribution (CC BY) license (https:/ / creativecommons.org/licenses/by/ $4.0 /)$.
1 Department of Nutrition, Food Science and Gastronomy, XaRTA, Institute of Nutrition and Food Safety (INSA-UB), School of Pharmacy and Food Sciences, University of Barcelona, 08028 Barcelona, Spain; julian.lozano@ub.edu (J.L.-C.); naye.yerena@gmail.com (A.L.-Y.); alexandra.olmo@ub.edu (A.O.-C.); mariaperez@ub.edu (M.P.); lamuela@ub.edu (R.M.L.-R.)

2 CIBER Physiopathology of Obesity and Nutrition (CIBEROBN), Institute of Health Carlos III, 28029 Madrid, Spain

3 Center of Scientific and Technological, University of Barcelona (CCiTUB), 08028 Barcelona, Spain; ojauregui@ccit.ub.edu

4 CIBER Fragilidad y Envejecimiento Saludable (CIBERfes), Instituto de Salud Carlos III, 18100 Barcelona, Spain

5 Laboratory of Organic Chemistry, Faculty of Pharmacy and Food Sciences, University of Barcelona, 08028 Barcelona, Spain

* Correspondence: avallverdu@ub.edu; Tel.: +34-934-024-508

+ These authors contributed equally to this work.

Abstract: Extra virgin olive oil (EVOO), one of the key foods of the Mediterranean diet, is distinguished by its high content of nutritional and antioxidant compounds compared to other vegetable oils. During EVOO production, the major secoiridoids of EVOO, oleacein, oleocanthal, ligstroside, and oleuropein aglycones, undergo a series of transformations to open- and closed-structure forms. The resulting mixture of compounds can become more complex during the analytical procedure, due to the keto-enol tautomerism of the open forms and their interaction with polar solvents, and therefore more challenging to analyze. Employing the same extraction method used to analyze the other EVOO phenolic compounds, we report here a simple UHPLC-ESI-MS/MS procedure for the quantification of those secoiridoids that is able to co-elute the different isomers of each compound. The method was validated following AOAC guidelines, and the matrix effect and recoveries were within satisfactory limits.

Keywords: EVOO analysis; polyphenols; Mediterranean diet; oleocanthal; oleacein; HPLC; mass spectrometry

\section{Introduction}

Extra virgin olive oil (EVOO) is the main fat of the Mediterranean Diet and its consumption is related to some health benefits as protection against cancer [1], type II diabetes [2], neurodegeneration [3], cardiovascular diseases, and reduces total mortality [4]. Those are attributed to its fatty acid profile, rich in mono-unsaturated fatty acids, and to its non-saponifiable fraction, rich in antioxidants [5]. The non-saponifiable fraction is composed by lipophilic compounds as vitamins, mainly $\alpha$-tocopherol; and carotenoids, mostly lutein and $\beta$-carotene; and a more hydrophilic molecules as phenolic compounds, above all, secoiridoids (SEC). This fraction, in addition to its health benefits, is the responsible of EVOO high appreciated taste. The volatiles compounds are responsible of its aroma, giving to it from notes of green fruit to notes of banana. Moreover, the phenolic compounds are well correlated with bitter, fruity, green, and pungent EVOO taste descriptors [6].

SEC represent 70-90\% of the total phenolic compounds in EVOO [7]. Among the major SEC in olive drupes are oleuropein and ligstroside [8,9], which evolve to their constituent 
aglycones of oleuropein (OLA) and ligstroside (LIG) during EVOO production. The hydrolyzation of glycosidic bonds during the crushing and malaxation processes leads to the formation of aglycones, primarily in the non-aldehydic closed form [10]. The initial aglycone compounds (closed form I) are then transformed by hydrolysis and keto-enol tautomerism into the open forms I and II (dialdehyde) and by a 1,4-Michael addition into the closed forms II and III (mono-aldehydes) [10,11]. These transformations can take place because the $\mathrm{pH}$ of the olive paste is approximately five and the compounds undergo an isomerization reaction with the acid in the water phase. Additionally, oleacein (OLE) and oleocanthal (OLC), also with open and closed forms, are generated through a decarboxylation step. The proposed processes of biotransformation and isomerization are depicted in Figure 1.
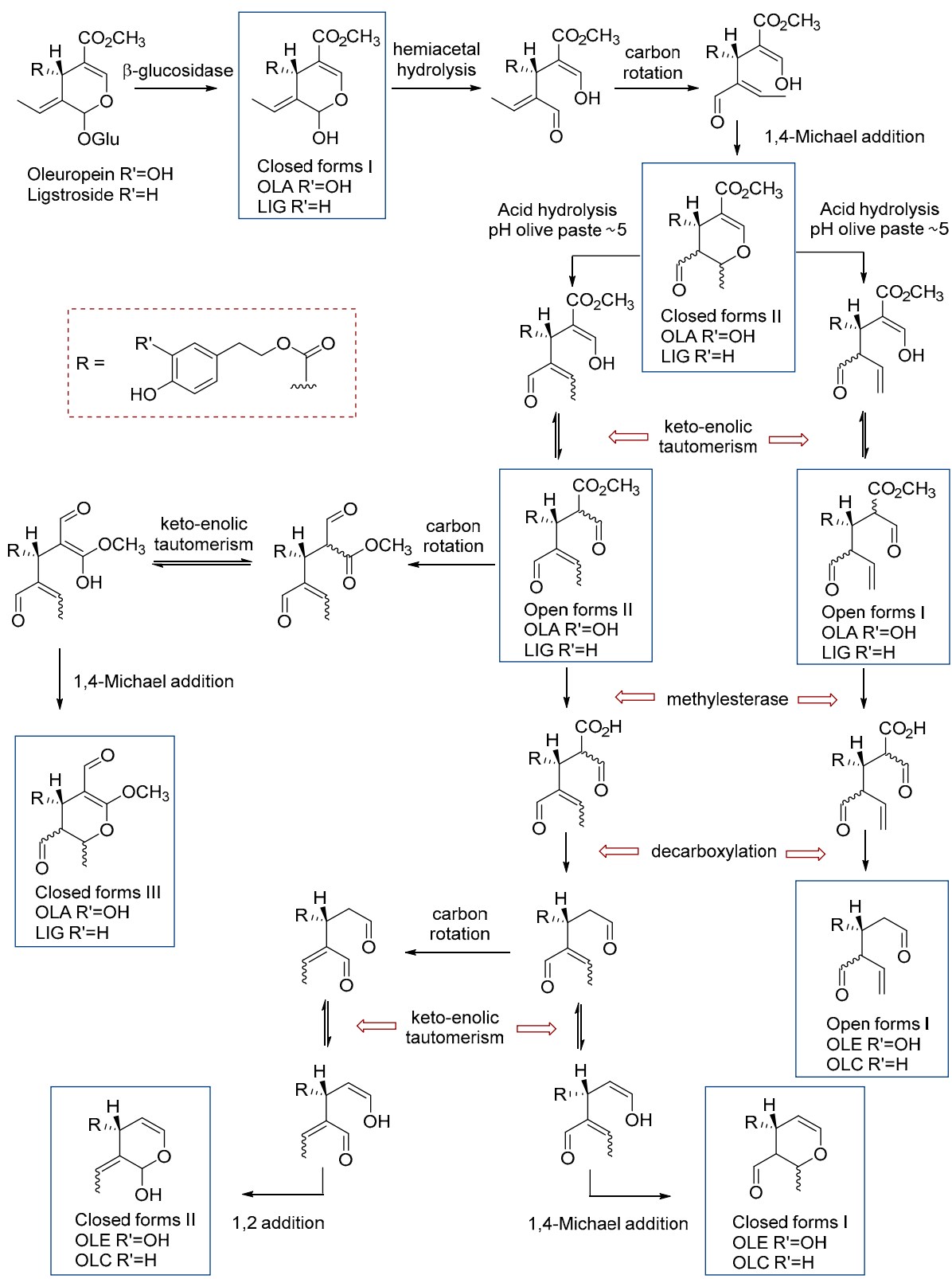

Figure 1. Conversion of Oleuropein and Ligstroside to oleuropein aglycone (OLA), oleacein (OLE), ligstroside aglycone (LIG), and oleocanthal (OLC) during oil extraction. Adapted from Abbattista et al., 2019 [10] and adding the closed forms I and II for OLC and OLE, which are possible artifacts.

Those phenolic compounds are the responsible of some EVOO health effects [12]. OLC is found to be a nonsteroidal anti-inflammatory compound, with effect similar to 
ibuprofen [13]. Furthermore, consumption of EVOO rich in OLC is associated with an anti-platelet effect, which may affect the prevention of cardiovascular diseases [14]. OLC also plays a remarkable role against neurogenerative diseases, primarily it reduces oxidative stress and protects against neuronal apoptosis [15]. It plays specific roles against Alzheimer's Disease, it inhibits $\beta$-amyloid formation, reduces its toxicity and enhances the clearance from the brain [16-18]. On the other hand, OLE prevents cardiovascular diseases by inhibiting LDL oxidation and preventing neutrophil adhesion $[19,20]$. OLE also has anti-cancer effect promoting apoptosis in leukemia and skin cancer cells [21,22]. LIG also has shown anti-cancer effect, against breast cancer cells inducing apoptosis [23] and inhibiting c-MET signaling and then having antimigratory activity [24]. Finally, OLA has proven to reduce the levels of total cholesterol, LDL, and triglycerides and to increase HDL and liver antioxidant enzymes in vivo [25].

SEC concentration in EVOO depends on different agronomical and technical factors. For instance, the variety is the most affecting factor [26]. For example, varieties such as the Spanish "Picual" or the Italian "Coratina" contain high concentrations in SEC [26,27]. Additionally, there are some Ivory-wild varieties that produce an EVOO very rich in SEC [28]. Another factor that affects SEC concentration is the maturity stage of the olives. The different enzymatic activities of the olive varieties cause the phenolic profile to vary distinctly during the ripening [27]. Moreover, technical factors also play an important role during EVOO extraction, an increase in the malaxation temperature will lead to an increase in enzymatic activity during EVOO extraction [29]. In addition, an increase in malaxation time will increase the time those enzymes are working, having more reaction from LIG and OLA to OLC and OLE, respectively, then increasing OLC and OLE concentration and decreasing LIG and OLA [30]. Finally, the type and diameter of sieve also affect the oil composition [31]. Thus, an accurate analysis of those compounds could shed light on how agronomical and technical factors affect the bioactive components of EVOO.

Different methods are available for the analysis of EVOO phenolic compounds [32,33], but liquid chromatography coupled to mass spectrometry (MS) has proven to be the best in terms of selectivity and limits of detection (LOD) and quantification (LOQ) [34]. Colorimetric methods have been well correlated with individual phenols, although they only give an idea of the total amount and not of the phenolic profile of the oil [35]. However, during the chromatographic analysis, the open-structure forms of the four main SEC of EVOO can undergo keto-enol tautomerism, and monohydrates, methyl hemiacetals and dimethyl acetals can be generated when the sample is prepared with water and methanol $(\mathrm{MeOH})$. Therefore, chromatographic analysis of those SEC has several impediments. In addition, the open-ring forms I and II (dialdehyde), closed form I (non-aldehyde) and closed forms II and III (mono-aldehyde) can be detected when OLA and LIG are in contact with a protic solvent such as water $[10,36,37]$. SEC can also form acetals and hemi-acetals with $\mathrm{MeOH}$ and water [38]. Curiously, the methyl hemi-acetal of OLC has been previously detected in EVOO but not identified, being reported as unknown [39].

The methods of Suarez et al. (2008) resulted in wide and jagged peaks for OLC and OLE [40]. To block the carbonylic species and avoid the tautomeric equilibrium and interaction with solvents, Di Donna et al. (2011) proposed carrying out in situ chemical derivatization of the aldehyde functions with methoxyamine before the analysis of OLC and OLE with high-performance liquid chromatography (HPLC) coupled with mass spectrometry in tandem (MS/MS), but this extra step resulted in a more complicated and expensive method [41]. Alternatively, Karkoula et al. (2012) developed a quantitative ${ }^{1} \mathrm{H}-\mathrm{NMR}$ method for the analysis of the dialdehyde forms of OLC and OLE [42], which was later extended to the analysis of the closed monoaldehyde forms of the aglycones [43]. However, a large amount of EVOO $(5 \mathrm{~g})$ and solvents $(50 \mathrm{~mL})$ are required to ensure a sufficient quantity of analytes in the sample, and the extract cannot be used for the analysis of the other phenolic compounds. Another disadvantage is that this method also needs an external calibration curve, as the extraction is not complete, requiring then large amounts of pure compounds, although this calibration curve is just needed once, as the NMR response 
is constant. Furthermore, the method cannot quantitate all isoforms of LIG and OLA, just the closed monoaldehyde forms. (closed forms II Figure 1). Diamantakos et al. (2015) determined that the ratio of the open dialdehyde forms and the open monoaldehyde form (the corresponding enolic form) was 1:1:2, respectively [37]. Later, the same authors used the 1:1:2 ratio to calculate also the concentration of these form integrating the enol peak of the open monoaldehyde form [44]. However, the reagent and time-consuming problem of the extraction required for the NMR analysis is not solved.

Sánchez de Medina et al. (2017) developed a method to determine OLC and OLE, but not the other EVOO SEC [38]. In addition, in later experiments, they also quantitate OLA and LIG, but without method validation [45]. Moreover, Celano et al. (2018) studied the SEC transformations during the extraction and chromatographic process and validated a method for the quantitation of SEC through ultra-high performance liquid chromatography (UHPLC) coupled to a UV detector. However, this approach is time-consuming (27 min) and the LOD and LOQ are high [46]. Finally, a direct method for the analysis of SEC by UHPLC coupled to MS/MS, minimizing the generation of acetals and hemiacetals, was recently validated. Nevertheless, a separate extraction process was required, as the extraction method proved unsuitable for the other phenolic compounds [47]. Furthermore, a ternary pump and a chromatographic instrument adequate for strong organic solvents, not always available, were also necessary.

Herein we report the development and validation of a simple analytical method based on UHPLC coupled with electrospray ionization and MS/MS (UHPLC-ESI-MS/MS) for the analysis of the four major SEC compounds in EVOO, able to coelute their isomers and achieve well-shaped peaks. Matrix-matched calibration was used, employing the same extraction process as for the other phenolic compounds in EVOO and a defatting step. The matrix effect of the oil and the analyte recoveries were also assessed.

\section{Materials and Methods}

\subsection{Reagents}

OLC ( $\geq 95 \%$ purity) was purchased from Merck (Darmstadt, Germany), and OLE ( $\geq 90 \%$ purity) and OLA ( $\geq 95 \%$ purity) from Toronto Research Chemicals (North York, $\mathrm{ON}$, Canada). $\mathrm{MeOH}$, acetonitrile (ACN) and formic acid were acquired from AppliChem, Panreac Quimica SLU (Barcelona, Spain). Hexane, 4-hydroxybenzaldehyde, chloroform- $d$, tetramethylsilane and gentisic acid were purchased from Sigma-Aldrich (St. Louis, MO, USA). 3-(4-hydroxy-3-methoxyphenyl)propionic acid was purchased from Fisher Scientific (Waltham, MA, USA) and cyclohexane from Carlo Erba (Madrid, Spain). Ultrapure water was obtained using a Milli-Q purification system (Millipore, Bedford, MA, USA).

\subsection{SEC Extraction for UHPLC-ESI-MS/MS Analysis}

SEC were extracted by a liquid-liquid extraction following the procedure proposed by Capriotti et al. (2014) [48]. Briefly, $0.5 \mathrm{~g}$ of EVOO was dissolved in $1 \mathrm{~mL}$ of hexane in a $10 \mathrm{~mL}$ centrifuge tube and shaken for $30 \mathrm{~s}$. The phenolic compounds were extracted as follows: $2 \mathrm{~mL}$ of a solution of MeOH:water $(4: 1 \mathrm{v} / \mathrm{v})$ was added to the tube and stirred for $30 \mathrm{~s}$. The resulting emulsion was centrifuged at $3000 \mathrm{rpm}$ and $4{ }^{\circ} \mathrm{C}$ for $3 \mathrm{~min}$. After separation of the hexane phase, the methanolic-aqueous extract was washed with hexane. To ensure the complete removal of SEC from the hydrophobic phase, a second extraction of phenolic compounds was carried out from the hexane phase with MeOH:water $(4: 1 v / v)$, using the aforementioned conditions. The combined methanolic-aqueous extracts were evaporated under a nitrogen curtain and reconstituted with $800 \mu \mathrm{L}$ of MeOH:water (80:20 $v / v)$, filtered with polytetrafluoroethylene syringe filters $(0.2 \mu \mathrm{m})$, transferred to an amber glass vial, and stored at $-80{ }^{\circ} \mathrm{C}$ until analysis. All procedure was done under UV-filtered light to avoid SEC photooxidation.

The calibration curve was prepared in refined olive oil, which was spiked with the standards before extraction at the following concentrations: 1, 2, 5, 8, 10, and $20 \mathrm{mg} \cdot \mathrm{kg}^{-1}$. 


\subsection{UHPLC-ESI-MS/MS Analysis}

The analysis was carried out using a UHPLC Acquity system equipped with a binary pump and autosampler (Waters, Milford, MA, USA), coupled to an API3000 triple quadrupole mass spectrometer (ABSciex, Framingham, MA, USA) equipped with a TurboIonspray source operating in negative mode. An Acquity UPLC ${ }^{\circledR}$ BEH C18 column $(2.1 \times 50 \mathrm{~mm}$, i.d., $1.7 \mu \mathrm{m}$ particle size $)$ with an Acquity UPLC ${ }^{\circledR}$ BEH C18 pre-column $\left(2.1 \times 5 \mathrm{~mm}\right.$, i.d., $1.7 \mu \mathrm{m}$ particle size) (Waters Corporation ${ }^{\circledR}$, Dublin, Ireland) were used for the main SEC separation. The mobile phases consisted of $\mathrm{MeOH}$ with $0.1 \%$ of formic acid (A) and water with $0.1 \%$ of formic acid (B) and the flow was constant at a rate $0.6 \mathrm{~mL} \cdot \mathrm{min}$. The gradient was as follows: $\mathrm{t}=0 \mathrm{~min}, 100 \%(\mathrm{~A}) ; \mathrm{t}=2 \mathrm{~min}, 100 \%(\mathrm{~A})$; $\mathrm{t}=4.75 \mathrm{~min}, 46.4 \%(\mathrm{~A}) ; \mathrm{t}=4.9 \mathrm{~min}, 0 \%(\mathrm{~A}) ; \mathrm{t}=5.9 \mathrm{~min}, 0 \%(\mathrm{~A}) ; \mathrm{t}=6 \mathrm{~min}, 100 \%(\mathrm{~A})$; $\mathrm{t}=6.5 \mathrm{~min}, 100 \%$ (A). The injection volume and column temperature were $5 \mu \mathrm{L}$ and $50{ }^{\circ} \mathrm{C}$, respectively.

Ionization, in negative mode, was achieved using electrospray ionization (ESI) and all the compounds were monitored in the multiple monitoring mode (MRM) with the following settings: capillary voltage, $-4000 \mathrm{~V}$; nebulizer gas $\left(\mathrm{N}_{2}\right), 10$ (arbitrary units); curtain gas $\left(\mathrm{N}_{2}\right), 12$ (arbitrary units); and drying gas $\left(\mathrm{N}_{2}\right)$ heated to $450{ }^{\circ} \mathrm{C}$ at flow rate $8000 \mathrm{c} / \mathrm{min}$. The declustering potential, focusing potential, collision energy and entrance potential are shown in Table 1. The system was controlled by Analyst version 1.4.2 software supplied by ABSciex, the chromatograms were integrated with this same software.

Table 1. Electrospray ionization parameters for the secoiridoids (SEC).

\begin{tabular}{ccccc}
\hline & OLE & OLC & OLA & LIG \\
\hline MRM Transition & $361 / 291$ & $303 / 285$ & $377 / 275$ & $319 / 69$ \\
Declustering Potential & -40 & -40 & -45 & -30 \\
Focusing Potential & -170 & -170 & -140 & -170 \\
Entrance Potential & -5 & -5 & -5 & -5 \\
Collision Energy & -10 & -10 & -15 & -30 \\
Dwell Time (ms) & 150 & 50 & 150 & 50 \\
Retention time (min) & 2.7 & 2.4 & 2.5 & 2.3 \\
\hline
\end{tabular}

\subsection{Validation of the UHPLC-ESI-MS/MS Method}

The method was validated following the AOAC guidelines for dietary supplements and botanicals [49]. A matrix-matched calibration was selected, as previous experiments show low analyte recoveries [40]. The standards used were OLC, OLE, and OLA, but not LIG, as it is not commercially available. Selectivity, linearity, accuracy, repeatability, the carry-over between samples and the LOD and LOQ were tested. Regarding the selectivity, the ability to distinguish the analyte from other substances was indicated by an absence of the respective peaks at the same retention time as the corresponding standards in trace chromatograms obtained in multiple monitoring mode.

For assessing the cleaning step of the chromatographic method, the carry-over was evaluated. The carry-over is the increment of the signal of a peak due to residual analyte from preceding sample that remains in the instrument [50]. As AOAC guidelines do not have a protocol to evaluate the carry-over, the guideline on bioanalytical method validation from the European medicines agency was followed [50]. Hence, a blank sample was injected after the highest peak of the calibration curve $\left(20 \mathrm{mg} \cdot \mathrm{kg}^{-1}\right)$ and the carry-over should not be greater than $20 \%$ of the LOQ.

The linearity was evaluated by developing calibration curves using refined olive oil spiked with the analytes before the extraction, with 6 concentration points ranging from 1 to $20 \mathrm{mg} \cdot \mathrm{kg}^{-1}$ and five replicates for each point. The residuals were then tested through a residual plot. The $\mathrm{R}^{2}$ was also calculated, as were the accuracies of each point of the curve, based on the ratio between the calculated concentration and the spiked concentration; following AOAC guidelines, accuracies between 80 and 120\% were accepted as valid. When necessary, weighted regression was applied to improve the accuracy [51]. 
For calculating the curves with weighting, and without it, and for the residual plot test $\mathrm{R}$ version 4.0.3 (The $\mathrm{R}$ foundation) with RStudio version 1.3.1 (Rstudio, PBC) software was used. For the rest of the calculus, Excel 2017 (Microsoft SA) software was used.

To determine the repeatability of the method, both intra- and inter-day repeatability were tested. Five replicates of a low-, intermediate- and upper-point of the curve $(1,5$, and $10 \mathrm{mg} \cdot \mathrm{kg}^{-1}$ ) were injected three times on the same day for the intra-day and three times on three different days for the inter-day repeatability. The relative standard deviations (RSD) for the inter- and intra-day experiments were calculated. All the values lower than $\mathrm{RSD}=$ Concentration $^{-(0.15)}$ were accepted. Those values are lower than $8 \%$ for $1 \mathrm{mg} \cdot \mathrm{kg}^{-1}$, $7 \%$ for $5 \mathrm{mg} \cdot \mathrm{kg}^{-1}$ and $6 \%$ for $10 \mathrm{mg} \cdot \mathrm{kg}^{-1}$.

The LOD is the smallest concentration of the analyte that can be distinguished from the blank and by definition is 3 times the standard deviation of the blank. The LOQ is the smallest amount or concentration of an analyte that can be determined with acceptable reliability and corresponds to 10 times the standard deviation of the blank. The LOD and LOQ were determined using the lower point of the curve, calculating the signal-to-noise ratio of that point, and extrapolating the concentration with a signal-to-noise ratio of 3 and 10 , respectively. Then each analyte was prepared at the LOD and LOQ concentration to verify the obtained value.

\subsection{Matrix Effect and Recovery}

The matrix effect and the recovery of the developed method were assessed following the procedure of Matuszewski et al. (2003) [52]. The matrix effect is the change of the analyte signal due to coeluted substances, whilst the recovery is the percentage of the analyte that remains after the extraction. Three different calibration curves were prepared, two of them using refined olive oil spiked with the standard SEC compounds before and after the extraction process, named "spiked-before" and "spiked-after" curves (SBC and SAC, respectively). The third curve was generated by directly diluting the standards in the reconstitution phase, namely, the reconstitution phase curve (RPC). By comparing the SBC and SAC, it is possible to evaluate the recovery of the extraction. Whereas the matrix effect can be assessed by comparing the SAC and RPC, as the interferences form the matrix are not present in the RPC.

\section{Results and Discussion}

\subsection{Analytical Method Development}

The chromatographic conditions were established as in a previous study [38]. Different percentages of formic acid (from $0.02 \%$ to $1 \%$ ) in both mobile phases were tested to improve the peak shape and ionization of the SEC. The best ionizations were achieved with $0.1 \%$ of formic acid in both water and $\mathrm{MeOH}$. The ionization parameters were then optimized for each compound to obtain the highest signal through direct infusion of the analytes at $1 \mathrm{mg} \cdot \mathrm{L}^{-1}$. The parameters optimized were the declustering potential, focusing potential, collision energy, and entrance potential. Finally, the dwell time was optimized to obtain a smooth peak. The results are shown in Table 1.

As already mentioned, the coexistence of open- and closed-structure forms of SEC compounds hinders their chromatographic analysis. However, we improved the gradient to minimize the number of peaks for each compound and obtained thin and symmetric peaks. These new chromatographic conditions allowed us to coelute the different isomers together, all of which could thus be quantified at the same time. An example of a chromatogram for each analyte is shown in Figure 2. OLE and OLC isoforms were eluted in one peak, whereas OLA and LIG still produced more than one, although with acceptable accuracy.

Finally, when analyzing oil samples with a reverse phase column, sometimes the chromatographic conditions cannot properly clean the column and cross-contamination of samples can occur. To prevent this, Luque et al. (2019) implemented a cleaning step based on ACN and tetrahydrofuran, requiring a ternary pump system and an HPLC 
instrument prepared for strong solvents, which are not always available [47]. In our study, the chromatographic cleaning step was confirmed by testing the carry-over in a blank sample injected right after the most concentrated point of the calibration curve $\left(20 \mathrm{mg} \cdot \mathrm{kg}^{-1}\right)$. The carry-over for OLE was null, in the case of OLC and OLA, despite being a small peak, it was less than $20 \%$ of the LOQ. The chromatograms are showed in Figure S1. As the extraction method includes a defatting step with hexane, the use of strong organic solvents was not needed to avoid carry-over.

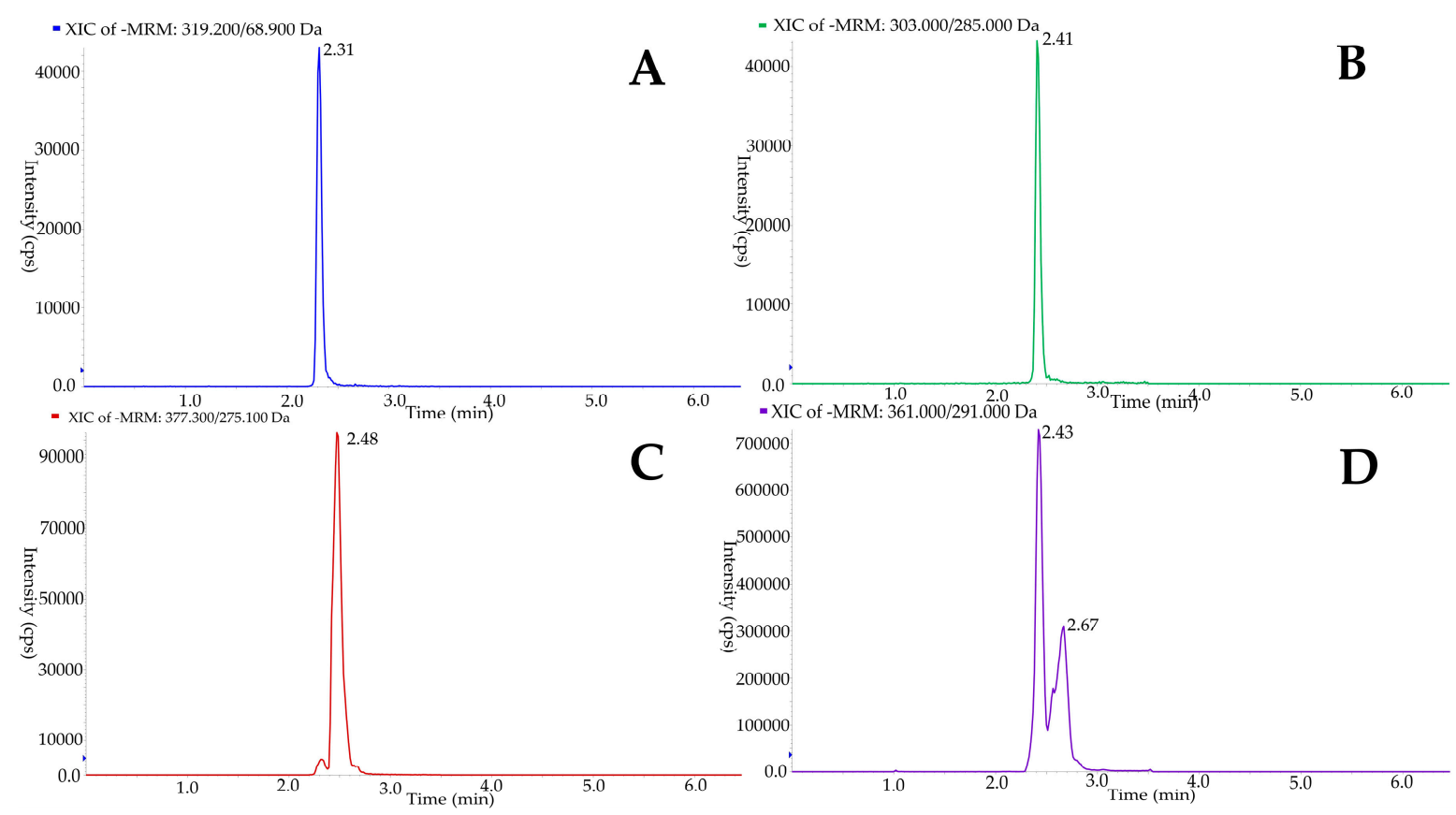

Figure 2. Example chromatogram of oleacein (A), oleocanthal (B), oleuropein aglycone (C), and ligstroside aglycone (D), the latter from a commercial "Picual" EVOO.

Additionally, the use of an internal standard was tested to minimize errors. The best internal standards for a MS quantitation method are compound analogues isotopically labeled with deuterium (D) or ${ }^{13} \mathrm{C}$, but OLA, LIG, OLE, and OLC derivatives are not commercially available. Therefore, compounds structurally similar to our target analytes were tested as internal standards, such as gentisic acid and 3-(4-hydroxy-3-methoxyphenyl)propionic acid. Those were tested at $5 \mathrm{mg} \cdot \mathrm{kg}^{-1}$, corresponding to an intermediate concentration of the calibration curve. However, the resulting accuracies were lower than without the use of internal standards.

\subsection{Analytical Method Validation}

The method was assessed in terms of linearity, selectivity, accuracy, repeatability, and LOD and LOQ (results shown in Table 2). The selectivity was visually checked in the chromatograms, which showed no other analytes except those of interest.

The linearity range was examined visually in the residual plot. In the case of OLC and OLA, a $1 / x^{2}$ and $1 / x$ weighting was used, respectively, to improve the accuracy for the low concentration points [51]. Then, as the curve was built with a weighted least squares method, the linearity was checked through the standardized residual plots (depicted in Figure S2). All the three analytes (OLC, OLE, and OLA) showed linearity in the tested range between 1 and $20 \mathrm{mg} \cdot \mathrm{kg}^{-1}$. Other studies report linearity from 3 to $200 \mu \mathrm{g} \cdot \mathrm{kg}^{-1}$ for OLE and LIG, from 1 to $100 \mu \mathrm{g} \cdot \mathrm{kg}^{-1}$ for OLA, and from 12 to $800 \mu \mathrm{g} \cdot \mathrm{kg}^{-1}$ for OLC [47]. However, those margins are far below the usual concentration of these compounds in EVOO [45]. 
Table 2. Method validation parameters for OLE, OLC, and OLA.

\begin{tabular}{ccccc}
\hline & & OLE & OLC & OLA \\
\hline \multirow{3}{*}{ Calibration curve } & Slope & 27,987 & 33,800 & 82,737 \\
& Interception & $-19,765$ & -7875 & $-74,897$ \\
& $\mathrm{R}^{2}$ & 0.9991 & 0.9873 & 0.9907 \\
& Weighting & none & $1 / \mathrm{x}^{2}$ & $1 / \mathrm{x}$ \\
\hline \multirow{2}{*}{ Limits } & $\mathrm{LOD} \mathrm{mg} \cdot \mathrm{kg}^{-1}$ ) & 0.0343 & 0.0186 & 0.0334 \\
& $\mathrm{LOQ}\left(\mathrm{mg} \cdot \mathrm{kg}^{-1}\right)$ & 0.114 & 0.0621 & 0.111 \\
\hline \multirow{5}{*}{ Accuracies } & $1 \mathrm{mg} \cdot \mathrm{kg}^{-1}$ & $93 \%$ & $107 \%$ & $87 \%$ \\
& $2 \mathrm{mg} \cdot \mathrm{kg}^{-1}$ & $100 \%$ & $89 \%$ & $101 \%$ \\
& $5 \mathrm{mg} \cdot \mathrm{kg}^{-1}$ & $103 \%$ & $97 \%$ & $113 \%$ \\
& $8 \mathrm{mg} \cdot \mathrm{kg}^{-1}$ & $96 \%$ & $95 \%$ & $98 \%$ \\
& $10 \mathrm{mg} \cdot \mathrm{kg}^{-1}$ & $102 \%$ & $100 \%$ & $110 \%$ \\
& $20 \mathrm{mg} \cdot \mathrm{kg}^{-1}$ & $99 \%$ & $114 \%$ & $94 \%$ \\
\hline \multirow{3}{*}{ Repeatability Relative } & Intraday $1 \mathrm{mg} \cdot \mathrm{kg}^{-1}$ & $2.3 \%$ & $2.5 \%$ & $5.8 \%$ \\
Standard Deviation & Intraday $5 \mathrm{mg} \cdot \mathrm{kg}^{-1}$ & $1.9 \%$ & $2.7 \%$ & $1.8 \%$ \\
& Interday $1 \mathrm{mg} \cdot \mathrm{kg}^{-1} \cdot \mathrm{kg}^{-1}$ & $1.1 \%$ & $5.7 \%$ & $3.7 \%$ \\
& Interday $5 \mathrm{mg} \cdot \mathrm{kg}^{-1}$ & $2.4 \%$ & $3.3 \%$ & $7.5 \%$ \\
& Interday $10 \mathrm{mg} \cdot \mathrm{kg}^{-1}$ & $1.2 \%$ & $4.1 \%$ & $7.0 \%$ \\
& & & $6.0 \%$ & $3.1 \%$ \\
\hline
\end{tabular}

The LODs were in the order of $20 \mu \mathrm{g} \cdot \mathrm{kg}^{-1}$ and LOQs approximately $100 \mu \mathrm{g} \cdot \mathrm{kg}^{-1}$, which are sufficiently low to quantitate the SEC in EVOO [45]. The limits are higher than those reported by Luque et al. who reported a LOD of about $1 \mu \mathrm{g} \cdot \mathrm{kg}^{-1}$ and LOQ of about $3 \mu \mathrm{g} \cdot \mathrm{kg}^{-1}$, with even lower values for OLA $\left(0.3 \mu \mathrm{g} \cdot \mathrm{kg}^{-1}\right.$ and $0.9 \mu \mathrm{g} \cdot \mathrm{kg}^{-1}$, respectively) [47]. An explanation for these different results is that Luque-Muñoz et al. were using a more sensitive mass spectrometer (a Xevo TQS tandem quadrupole mass spectrometer from Waters) than the one used in this work. However, the LOD and LOQ of our method were far lower than those achieved with the ${ }^{1} \mathrm{H}-\mathrm{NMR}$ procedure published by Karkoula et al. (2014), in which the LOD was $1 \mathrm{mg} \cdot \mathrm{kg}^{-1}$ and the LOQ was $10 \mathrm{mg} \cdot \mathrm{kg}^{-1}$ for all compounds [43]. These high values are due to the analytical technique, as NMR is less sensitive than MS [53]. The LOD and LOQ obtained here are also lower than those reported by Suarez et al. [40]. Further comparison between methods is summarized in Table 3.

Table 3. Parameter comparison between methods for oleacein (OLE), oleocanthal (OLC) and oleuropein aglycone (OLA).

\begin{tabular}{|c|c|c|c|c|c|c|c|c|}
\hline & $\begin{array}{c}\text { LOD } \\
\left(\mathrm{mg} \cdot \mathrm{kg}^{-1}\right)\end{array}$ & $\begin{array}{c}\text { LOQ } \\
\left(\mathrm{mg} \cdot \mathrm{kg}^{-1}\right)\end{array}$ & $\mathbf{R}^{2}$ & $\begin{array}{l}\text { RSD } \\
(\%)^{a}\end{array}$ & $\begin{array}{c}\text { EVOO } \\
(\mathrm{g})\end{array}$ & Instrument & $\begin{array}{c}\text { Time of } \\
\text { Analysis (min) }\end{array}$ & Study \\
\hline \multirow{5}{*}{ OLE } & 0.0343 & 0.114 & 0.999 & 1.8 & 0.5 & UHPLC-ESI-MS/MS & 6.5 & Current study \\
\hline & 0.128 & 0.428 & - & 3.1 & 45 & UPLC-ESI-MS/MS & 40 & {$[40]$} \\
\hline & 0.002 & 0.005 & 0.999 & 10 & 1 & HPLC-ESI-MS/MS & 10 & [38] \\
\hline & 0.001 & 0.0033 & 0.995 & 1.9 & 1 & UHPLC-ESI-MS/MS ${ }^{b}$ & 7.5 & [47] \\
\hline & 1 & 10 & 0.994 & 4.3 & 5 & NMR & - & [42] \\
\hline \multirow{5}{*}{ OLC } & 0.0186 & 0.0621 & 0.987 & 3.6 & 0.5 & UHPLC-ESI-MS/MS & 6.5 & Current study \\
\hline & 0.072 & 0.244 & - & 3 & 45 & UPLC-ESI-MS/MS & 40 & [40] \\
\hline & 0.004 & 0.01 & 0.992 & 11 & 1 & HPLC-ESI-MS/MS & 10 & [38] \\
\hline & 0.004 & 0.012 & 0.994 & 4.4 & 1 & UHPLC-ESI-MS/MS ${ }^{\text {b }}$ & 7.5 & [47] \\
\hline & 1 & 10 & 0.999 & 4.3 & 5 & NMR & - & [42] \\
\hline \multirow{3}{*}{ OLA } & 0.0334 & 0.111 & 0.991 & 3.8 & 0.5 & UPLC-ESI-MS/MS & 6.5 & Current study \\
\hline & 0.0003 & 0.0009 & 0.996 & 2.5 & 1 & UHPLC-ESI-MS/MS ${ }^{\text {b }}$ & 7.5 & [47] \\
\hline & 1 & 10 & 0.999 & 4.1 & 5 & NMR & - & [43] \\
\hline
\end{tabular}

The parameters were chosen according to the criteria of the authors to help choose the most suitable analytical method. ${ }^{a}$ Relative standard deviation within the same day of analysis, when it was calculated for more than one concentration; the mean of the different RSD is showed in the table. ${ }^{b}$ The chromatographic instrument requires a ternary pump and to be prepared for strong organic solvents. 
The accuracy of the method was within the accepted limits of the AOAC guidelines (80-120\%) for all the concentrations and analytes. Other studies report similar results: the accuracies found by Luque et al. are all less than 15\% and those of Karkoula et al. (2012) are less than $16 \%[42,47]$.

Lastly, both the intra and inter-day repeatability of the method were within the accepted margins established by AOAC guidelines, which stipulate that the maximum RSD should be $6 \%$ for $10 \mathrm{mg} \cdot \mathrm{kg}^{-1}, 7 \%$ for $5 \mathrm{mg} \cdot \mathrm{kg}^{-1}$ and $8 \%$ for $1 \mathrm{mg} \cdot \mathrm{kg}^{-1}$. Similarly, Luque et al. reported an RSD between 2.5 and $8.5 \%$ for OLC at $594 \mathrm{ng} \cdot \mathrm{mL}^{-1}$ and $24.75 \mathrm{ng} \cdot \mathrm{mL}^{-1}$, respectively [47]. In contrast, Sanchez de Medina et al. showed higher deviations, being 10\% for OLC and 11\% for OLE, although in this case the concentrations were not specified [38].

\subsection{Matrix Effect and Recovery}

Table 4 shows the results of the matrix effect and the recoveries for each concentration of the curves and compound, calculated as described above. The recoveries for the phenolic extractions were partial and varied according to the compound and concentration, ranging between 50 and 100\%. Suarez et al. also found quite low recoveries for OLC (67\%) and OLE (71\%) [40].

Table 4. Matrix effect and recoveries for OLE, OLC, and OLA.

\begin{tabular}{lcccc}
\hline & OLE & OLC & OLA \\
\hline & $1 \mathrm{mg} \cdot \mathrm{kg}^{-1}$ & 56 & 93 & 20 \\
& $2 \mathrm{mg} \cdot \mathrm{kg}^{-1}$ & 50 & 76 & 27 \\
& $5 \mathrm{mg} \cdot \mathrm{kg}^{-1}$ & 86 & 129 & 57 \\
& $8 \mathrm{mg} \cdot \mathrm{kg}^{-1}$ & 79 & 123 & 51 \\
& $10 \mathrm{mg} \cdot \mathrm{kg}^{-1}$ & 82 & 134 & 56 \\
& $20 \mathrm{mg} \cdot \mathrm{kg}^{-1}$ & 93 & 152 & 69 \\
& $1 \mathrm{mg} \cdot \mathrm{kg}^{-1}$ & 59 & 66 \\
& $2 \mathrm{mg} \cdot \mathrm{kg}^{-1}$ & 96 & 86 \\
& $5 \mathrm{mg} \cdot \mathrm{kg}^{-1}$ & 98 & 61 & 74 \\
& $8 \mathrm{mg} \cdot \mathrm{kg}^{-1}$ & 80 & 62 & 106 \\
& $10 \mathrm{mg} \cdot \mathrm{kg}^{-1}$ & 96 & 61 & 88 \\
& $20 \mathrm{mg} \cdot \mathrm{kg}^{-1}$ & 89 & 60 & 93 \\
\hline
\end{tabular}

Matrix effect was observed due to the substances coextracted and coeluted in the chromatography with the phenols. In the case of OLE and OLA, the matrix effect was less than $100 \%$, indicating matrix suppression; the values ranged from 50 to $90 \%$ for OLE and 20 to $69 \%$ for OLA. On the contrary, matrix enhancing was observed for OLC, with values ranging from 75 to $150 \%$.

To minimize the error due to the matrix effect and recoveries, internal standard method, or matrix-matched method are suitable, both make the matrix effect and the recoveries the same for the curve and the samples, although the internal standard method does not need a white matrix. In this study a matrix-matched external calibration was carried out to validate the method, it was possible to use this calibration method as the refined olive oil served as a blank matrix.

\section{Conclusions}

Simple, quick and with reasonable cost analytical methods are needed for the better understanding of our world. In this context, this publication presents a suitable method for the analysis of EVOO SEC, whose analysis has been a challenge since their characterization [54]. The best methods up to now were NMR methods, which despite presenting a lot of advantages, require a high time- and reagent-consuming extraction of the samples. Furthermore, there is an UHPLC validated method for EVOO SEC analysis, which uses a non-generic UHPLC and requires a different sample extraction than the one used for other EVOO phenolic compounds. Therefore, in this work, a quick (6.5 min) chromato- 
graphic method was developed and validated for the analysis of the main SEC compounds in EVOO, using the same extraction procedure employed for the other EVOO phenolic compounds, rendering it low time- and reagent-consuming. The linearity of the method was between 1 and $20 \mathrm{mg} \cdot \mathrm{kg}^{-1}$, the accuracies were between AOAC accepted margins, and the LOD and LOQ relatively low (in the order of 20 and $100 \mu \mathrm{g} \cdot \mathrm{kg}^{-1}$, respectively). The cleaning step of the chromatographic method was good as the carry-over was null. This method would provide new tools for a better understanding on how EVOO phenolic profile changes during extraction and due to agronomical conditions, such as olive variety or olive maturity stage. Finally, it would help future research to determine better the effect of EVOO phenolic profile on animal and human health.

Supplementary Materials: The following are available online at https:/ / www.mdpi.com/article/10 .3390/antiox10040540/s1, Figure S1: Chromatograms of a water injection after a sample. Figure S2: Residual plot of the different calibration curves.

Author Contributions: Conceptualization, J.L.-C., A.L.-Y. and R.M.L.-R.; methodology, O.J., M.P. and A.V.-Q.; validation, O.J., M.P. and A.V.-Q.; formal analysis, J.L.-C. and A.L.-Y.; investigation, J.L.-C., A.L.-Y. and A.O.-C.; resources, R.M.L.-R.; data curation, J.L.-C., A.L.-Y. and A.O.-C.; writing-original draft preparation, J.L.-C. and A.L.-Y.; writing-review and editing, A.O.-C., O.J., M.P., R.M.L.-R. and A.V.-Q.; visualization, A.L.-Y.; supervision, R.M.L.-R. and A.V.-Q.; project administration, R.M.L.-R. and A.V.-Q.; funding acquisition, R.M.L.-R. All authors have read and agreed to the published version of the manuscript.

Funding: This research was funded by CICYT (AGL2016-75329-R), CIBEROBN from the Instituto de Salud Carlos III, ISCIII from the Ministerio de Ciencia, Innovación y Universidades, (AEI/FEDER, UE) and Generalitat de Catalunya (GC) (2017SGR 196). Julián Lozano-Castellón thanks the Ministry of Science Innovation and Universities for the FPI contract (BES-2017-080017). Anallely López-Yerena wishes to thank the Consejo Nacional de Ciencia y Tecnología (CONACYT) of Mexico for the doctoral scholarship. A.O.C. thanks the Ministerio de Ciencia, Innovación y Universidades for the FPU contract [FPU2018/03119]. Anna Vallverdú-Queralt thanks the Ministry of Science Innovation and Universities for the Ramon y Cajal contract (RYC-2016-19355).

Institutional Review Board Statement: Not applicable.

Informed Consent Statement: Not applicable.

Data Availability Statement: Data is contained within the article and Supplementary Material.

Acknowledgments: The authors wish to thank the CCiT-UB for the mass spectrometry and NMR equipment.

Conflicts of Interest: Rosa María Lamuela-Raventós reports receiving lecture fees from Cerveceros de España and receiving lecture fees and travel support from Adventia. The other authors declare no conflict of interest.

\begin{abstract}
Abbreviations
ACN: acetonitrile; ESI: Electrospray ionization; EVOO: Extra-virgin olive oil, HPLC: high-performance liquid chromatography; LIG: ligstroside aglycone; LOD: limit of detection; LOQ: limit of quantification; MeOH: methanol; MRM: multiple monitoring mode; MS: mass spectrometry; MS/MS: mass spectrometry in tandem; OLA: oleuropein aglycone; OLC: oleocanthal; OLE: oleacein; RPC: reconstitution phase curve; RSD: relative standard deviation; SAC: spiked-after curve; SBC: spiked-before curve; SEC: secoiridoids; UHPLC: ultra-high performance liquid chromatography; UV: ultraviolet.
\end{abstract}

\title{
References
}

1. Gotsis, E.; Anagnostis, P.; Mariolis, A.; Vlachou, A.; Katsiki, N.; Karagiannis, A. Health benefits of the mediterranean diet: An update of research over the last 5 years. Angiology 2015, 66, 304-318. [CrossRef]

2. Martínez-González, M.A.; Corella, D.; Salas-Salvadó, J.; Estruch, R.; Ros, E. Prevention of Diabetes With Mediterranean Diets. Ann. Intern. Med. 2014, 161, 157. [CrossRef] 
3. Martínez-Lapiscina, E.H.; Clavero, P.; Toledo, E.; Estruch, R.; Salas-Salvadó, J.; San Julián, B.; Sanchez-Tainta, A.; Ros, E.; Valls-Pedret, C.; Martinez-Gonzalez, M.Á. Mediterranean diet improves cognition: The PREDIMED-NAVARRA randomised trial. J. Neurol. Neurosurg. Psychiatry 2013, 84, 1318-1325. [CrossRef] [PubMed]

4. Guasch-Ferré, M.; Hu, F.B.; Martínez-González, M.A.; Fitó, M.; Bulló, M.; Estruch, R.; Ros, E.; Corella, D.; Recondo, J.; GómezGracia, E.; et al. Olive oil intake and risk of cardiovascular disease and mortality in the PREDIMED Study. BMC Med. 2014, 12, 12-78. [CrossRef] [PubMed]

5. Yubero-serrano, E.M.; Lopez-moreno, J.; Gomez-delgado, F.; Lopez-miranda, J. Extra virgin olive oil: More than a healthy fat. Eur. J. Clin. Nutr. 2019, 72, 8-17. [CrossRef] [PubMed]

6. Prata, R.; Pereira, J.A.; Rodrigues, N.; Dias, L.G.; Veloso, A.C.A.; Casal, S.; Peres, A.M. Olive Oil Total Phenolic Contents and Sensory Sensations Trends during Oven and Microwave Heating Processes and Their Discrimination Using an Electronic Tongue. J. Food Qual. 2018, 2018, 1-10. [CrossRef]

7. López-Yerena, A.; Lozano-Castellón, J.; Olmo-Cunillera, A.; Tresserra-Rimbau, A.; Quifer-Rada, P.; Jiménez, B.; Pérez, M.; Vallverdú-Queralt, A. Effects of organic and conventional growing systems on the phenolic profile of extra-virgin olive oil. Molecules 2019, 24, 1986. [CrossRef]

8. Kanakis, P.; Termentzi, A.; Michel, T.; Gikas, E.; Halabalaki, M.; Skaltsounis, A.L. From olive drupes to olive OilAn HPLCorbitrap-based qualitative and quantitative exploration of olive key metabolites. Planta Med. 2013, 79, $1576-1587$.

9. Jerman Klen, T.; Golc Wondra, A.; Vrhovšek, U.; Mozetič Vodopivec, B. Phenolic Profiling of Olives and Olive Oil Process-Derived Matrices Using UPLC-DAD-ESI-QTOF-HRMS Analysis. J. Agric. Food Chem. 2015, 63, 3859-3872. [CrossRef]

10. Abbattista, R.; Losito, I.; De Ceglie, C.; Basile, G.; Calvano, C.D.; Palmisano, F.; Cataldi, T.R.I. Structural characterization of the ligstroside aglycone isoforms in virgin olive oils by liquid chromatography-high-resolution Fourier-transform mass spectrometry and H/Dexchange. J. Mass Spectrom. 2019, 54, 843-855. [CrossRef]

11. Pérez-Trujillo, M.; Gómez-Caravaca, A.M.; Segura-Carretero, A.; Fernández-Gutiérrez, A.; Parella, T. Separation and identification of phenolic compounds of extra virgin Olive Oil from Olea europaea L. by HPLC-DAD-SPE-NMR/MS. Identification of a new diastereoisomer of the aldehydic form of oleuropein aglycone. J. Agric. Food Chem. 2010, 58, 9129-9136. [CrossRef]

12. Lozano-Castellón, J.; López-Yerena, A.; Rinaldi de Alvarenga, J.F.; Romero del Castillo-Alba, J.; Vallverdú-Queralt, A.; EscribanoFerrer, E.; Lamuela-Raventós, R.M. Health-promoting properties of oleocanthal and oleacein: Two secoiridoids from extra-virgin olive oil. Crit. Rev. Food Sci. Nutr. 2020, 60, 2532-2548. [CrossRef]

13. Beauchamp, G.K.; Keast, R.S.J.; Morel, D.; Lin, J.; Pika, J.; Han, Q.; Lee, C.H.; Smith, A.B.; Breslin, P.A.S. Ibuprofen-like activity in extra-virgin olive oil. Nature 2005, 437, 45-46. [CrossRef] [PubMed]

14. Agrawal, K.; Melliou, E.; Li, X.; Pedersen, T.L.; Wang, S.C.; Magiatis, P.; Newman, J.W.; Holt, R.R. Oleocanthal-rich extra virgin olive oil demonstrates acute anti-platelet effects in healthy men in a randomized trial. J. Funct. Foods 2017, 36, 84-93. [CrossRef] [PubMed]

15. Mete, M.; Aydemir, I.; Unsal, U.U.; Collu, F.; Vatandas, G.; Gurcu, B.; Duransoy, Y.K.; Taneli, F.; Tuglu, M.I.; Selcuki, M. Neuroprotective effects of oleocanthal, a compound in virgin olive oil, in a rat model of traumatic brain injury. Turk. Neurosurg. 2018, 28, 858-865. [CrossRef]

16. Abuznait, A.H.; Qosa, H.; Busnena, B.A.; El Sayed, K.A.; Kaddoumi, A. Olive-oil-derived oleocanthal enhances $\beta$-amyloid clearance as a potential neuroprotective mechanism against Alzheimer's disease: In vitro and in vivo studies. ACS Chem. Neurosci. 2013, 4, 973-982. [CrossRef]

17. Khanfar, M.A.; Bardaweel, S.K.; Akl, M.R.; El Sayed, K.A. Olive Oil-derived Oleocanthal as Potent Inhibitor of Mammalian Target of Rapamycin: Biological Evaluation and Molecular Modeling Studies. Phyther. Res. 2015, 14, 871-882. [CrossRef]

18. Qosa, H.; Batarseh, Y.S.; Mohyeldin, M.M.; El Sayed, K.A.; Keller, J.N.; Kaddoumi, A. Oleocanthal Enhances Amyloid- $\beta$ Clearance from the Brains of TgSwDI Mice and in Vitro across a Human Blood-Brain Barrier Model. ACS Chem. Neurosci. 2015, 6, 1849-1859. [CrossRef]

19. Sindona, G.; Caruso, A.; Cozza, A.; Fiorentini, S.; Lorusso, B.; Marini, E.; Nardi, M.; Procopio, A.; Zicari, S. Anti-Inflammatory Effect of 3,4-DHPEA-EDA [2-(3,4 -Hydroxyphenyl) ethyl (3S, 4E)-4-Formyl-3-(2-Oxoethyl)Hex-4-Enoate] on Primary Human Vascular Endothelial Cells. Curr. Med. Chem. 2012, 19, 4006-4013. [CrossRef]

20. Czerwińska, M.E.; Kiss, A.K.; Naruszewicz, M. Inhibition of human neutrophils NEP activity, CD11b/CD18 expression and elastase release by 3,4-dihydroxyphenylethanol-elenolic acid dialdehyde, oleacein. Food Chem. 2014, 153, 1-8. [CrossRef]

21. Fabiani, R.; De Bartolomeo, A.; Rosignoli, P.; Servili, M.; Selvaggini, R.; Montedoro, G.F.; Di Saverio, C.; Morozzi, G. Virgin olive oil phenols inhibit proliferation of human promyelocytic leukemia cells (HL60) by inducing apoptosis and differentiation. J. Nutr. 2006, 136, 614-619. [CrossRef]

22. Polini, B.; Digiacomo, M.; Carpi, S.; Bertini, S.; Gado, F.; Saccomanni, G.; Macchia, M.; Nieri, P.; Manera, C.; Fogli, S. Oleocanthal and oleacein contribute to the in vitro therapeutic potential of extra virgin oil-derived extracts in non-melanoma skin cancer. Toxicol. In Vitro 2018, 52, 243-250. [CrossRef]

23. Menendez, J.A.; Vazquez-Martin, A.; Garcia-Villalba, R.; Carrasco-Pancorbo, A.; Oliveras-Ferraros, C.; Fernandez-Gutierrez, A.; Segura-Carretero, A. TabAnti-HER2 (erbB-2) oncogene effects of phenolic compounds directly isolated from commercial Extra-Virgin Olive Oil (EVOO). BMC Cancer 2008, 8, 377. [CrossRef]

24. Busnena, B.A.; Foudah, A.I.; Melancon, T.; El Sayed, K.A. Olive secoiridoids and semisynthetic bioisostere analogues for the control of metastatic breast cancer. Bioorg. Med. Chem. 2013, 21, 2117-2127. [CrossRef] [PubMed] 
25. Jemai, H.; Bouaziz, M.; Fki, I.; El Feki, A.; Sayadi, S. Hypolipidimic and antioxidant activities of oleuropein and its hydrolysis derivative-rich extracts from Chemlali olive leaves. Chem. Biol. Interact. 2008, 176, 88-98. [CrossRef]

26. Ceci, L.N.; Mattar, S.B.; Carelli, A.A. Chemical quality and oxidative stability of extra virgin olive oils from San Juan province (Argentina). Food Res. Int. 2017, 100, 764-770. [CrossRef]

27. López-Huertas, E.; Lozano-Sánchez, J.; Segura-Carretero, A. Olive oil varieties and ripening stages containing the antioxidants hydroxytyrosol and derivatives in compliance with EFSA health claim. Food Chem. 2021, 342, 128291. [CrossRef]

28. López-Yerena, A.; Ninot, A.; Lozano-Castellón, J.; Escribano-Ferrer, E.; Romero-Aroca, A.J.; Belaj, A.; Vallverdú-Queralt, A.; Lamuela-Raventós, R.M. Conservation of Native Wild Ivory-White Olives from the MEDES Islands Natural Reserve to Maintain Virgin Olive Oil Diversity. Antioxidants 2020, 9, 1009. [CrossRef]

29. Taticchi, A.; Esposto, S.; Veneziani, G.; Urbani, S.; Selvaggini, R.; Servili, M. The influence of the malaxation temperature on the activity of polyphenoloxidase and peroxidase and on the phenolic composition of virgin olive oil. Food Chem. 2013, 136, 975-983. [CrossRef] [PubMed]

30. Miho, H.; Moral, J.; López-González, M.A.; Díez, C.M.; Priego-Capote, F. The phenolic profile of virgin olive oil is influenced by malaxation conditions and determines the oxidative stability. Food Chem. 2020, 314, 126183. [CrossRef] [PubMed]

31. Polari, J.J.; Wang, S.C. Hammer Mill Sieve Design Impacts Olive Oil Minor Component Composition. Eur. J. Lipid Sci. Technol. 2019, 121, 1900168. [CrossRef]

32. Reboredo-Rodríguez, P.; Valli, E.; Bendini, A.; Di Lecce, G.; Simal-Gándara, J.; Gallina Toschi, T. A widely used spectrophotometric assay to quantify olive oil biophenols according to the health claim (EU Reg. 432/2012). Eur. J. Lipid Sci. Technol. 2016, 118, 1593-1599. [CrossRef]

33. Olmo-Cunillera, A.; López-Yerena, A.; Lozano-Castellón, J.; Tresserra-Rimbau, A.; Vallverdú-Queralt, A.; Pérez, M. NMR spectroscopy: A powerful tool for the analysis of polyphenols in extra virgin olive oil. J. Sci. Food Agric. 2020, 100, $1842-1851$. [CrossRef]

34. Olmo-García, L.; Fernández-Fernández, C.; Hidalgo, A.; Vílchez, P.; Fernández-Gutiérrez, A.; Marchal, R.; Carrasco-Pancorbo, A. Evaluating the reliability of specific and global methods to assess the phenolic content of virgin olive oil: Do they drive to equivalent results? J. Chromatogr. A 2019, 1585, 56-69. [CrossRef] [PubMed]

35. Alessandri, S.; Ieri, F.; Romani, A. Minor polar compounds in extra virgin olive oil: Correlation between HPLC-DAD-MS and the Folin-Ciocalteu spectrophotometric method. J. Agric. Food Chem. 2014, 62, 826-835. [CrossRef]

36. Corominas-Faja, B.; Santangelo, E.; Cuyàs, E.; Micol, V.; Joven, J.; Ariza, X.; Segura-Carretero, A.; García, J.; Menendez, J.A. Computer-aided discovery of biological activity spectra for anti-aging and anti-cancer olive oil oleuropeins. Aging (Albany NY) 2014, 6, 731-741. [CrossRef]

37. Diamantakos, P.; Velkou, A.; Killday, K.B.; Gimisis, T.; Melliou, E.; Magiatis, P. Oleokoronal and oleomissional: New major phenolic ingredients of extra virgin olive oil. Olivae 2015, 122, 22-33.

38. Sánchez de Medina, V.; Miho, H.; Melliou, E.; Magiatis, P.; Priego-Capote, F.; Luque de Castro, M.D. Quantitative method for determination of oleocanthal and oleacein in virgin olive oils by liquid chromatography-tandem mass spectrometry. Talanta 2017, 162, 24-31. [CrossRef] [PubMed]

39. Carrasco-Pancorbo, A.; Neusüß, C.; Pelzing, M.; Segura-Carretero, A.; Fernández-Gutiérrez, A. CE- and HPLC-TOF-MS for the characterization of phenolic compounds in olive oil. Electrophoresis 2007, 28, 806-821. [CrossRef]

40. Suárez, M.; Macià, A.; Romero, M.P.; Motilva, M.J. Improved liquid chromatography tandem mass spectrometry method for the determination of phenolic compounds in virgin olive oil. J. Chromatogr. A 2008, 1214, 90-99. [CrossRef]

41. Di Donna, L.; Benabdelkamel, H.; Mazzotti, F.; Napoli, A.; Nardi, M.; Sindona, G. High-throughput assay of oleopentanedialdheydes in extra virgin olive oil by the UHPLC-ESI-MS/MS and isotope dilution methods. Anal. Chem. 2011, 83, 1990-1995. [CrossRef]

42. Karkoula, E.; Skantzari, A.; Melliou, E.; Magiatis, P. Direct measurement of oleocanthal and oleacein levels in olive oil by quantitative $1 \mathrm{H}$ NMR. Establishment of a new index for the characterization of extra virgin olive oils. J. Agric. Food Chem. 2012, 60, 11696-11703. [CrossRef]

43. Karkoula, E.; Skantzari, A.; Melliou, E.; Magiatis, P. Quantitative measurement of major secoiridoid derivatives in olive oil using qNMR. Proof of the artificial formation of aldehydic oleuropein and ligstroside aglycon isomers. J. Agric. Food Chem. 2014, 62, 600-607. [CrossRef]

44. Diamantakos, P.; Giannara, T.; Skarkou, M.; Melliou, E.; Magiatis, P. Influence of Harvest Time and Malaxation Conditions on the Concentration of Individual Phenols in Extra Virgin Olive Oil Related to Its Healthy Properties. Molecules 2020, $25,2449$. [CrossRef] [PubMed]

45. Miho, H.; Díez, C.M.; Mena-Bravo, A.; Sánchez de Medina, V.; Moral, J.; Melliou, E.; Magiatis, P.; Rallo, L.; Barranco, D.; Priego-Capote, F. Cultivar influence on variability in olive oil phenolic profiles determined through an extensive germplasm survey. Food Chem. 2018, 266, 192-199. [CrossRef] [PubMed]

46. Celano, R.; Piccinelli, A.L.; Pugliese, A.; Carabetta, S.; Di Sanzo, R.; Rastrelli, L.; Russo, M. Insights into the Analysis of Phenolic Secoiridoids in Extra Virgin Olive Oil. J. Agric. Food Chem. 2018, 66, 6053-6063. [CrossRef] [PubMed]

47. Luque-Muñoz, A.; Tapia, R.; Haidour, A.; Justicia, J.; Cuerva, J.M. Direct determination of phenolic secoiridoids in olive oil by ultra-high performance liquid chromatography-triple quadruple mass spectrometry analysis. Sci. Rep. 2019, 9, 1-9. [CrossRef] 
48. Capriotti, A.L.; Cavaliere, C.; Crescenzi, C.; Foglia, P.; Nescatelli, R.; Samperi, R.; Laganà, A. Comparison of extraction methods for the identification and quantification of polyphenols in virgin olive oil by ultra-HPLC-QToF mass spectrometry. Food Chem. 2014, 158, 392-400. [CrossRef] [PubMed]

49. AOAC International. Appendix K: Guidelines for Dietary Supplements and Botanicals, Part 1 AOAC Guidelines for SingleLaboratory Validation of Chemical Methods for Dietary Supplements and Botanicals. 2013. Available online: https: / / members. aoac.org/AOAC_Docs/StandardsDevelopment/eoma_appendix_k.pdf (accessed on 10 May 2019).

50. European Medicines Agency. Guideline on Bioanalytical Method Validation. 2011. Available online: https://www.ema.europa. eu/en/documents/scientific-guideline/guideline-bioanalytical-method-validation_en.pdf (accessed on 10 May 2019).

51. Kiser, M.M.; Dolan, J.W. Selecting the best curve fit. LC-GC Eur. 2004, 17, 138-143.

52. Matuszewski, B.K.; Constanzer, M.L.; Chavez-Eng, C.M. Strategies for the assessment of matrix effect in quantitative bioanalytical methods based on HPLC-MS/MS. Anal. Chem. 2003, 75, 3019-3030. [CrossRef]

53. Emwas, A.H.M. The strengths and weaknesses of NMR spectroscopy and mass spectrometry with particular focus on metabolomics research. Methods Mol. Biol. 2015, 1277, 161-193. [PubMed]

54. Montedoro, G.; Servili, M.; Baldioli, M.; Miniati, E. Simple and hydrolyzable phenolic compounds in virgin olive oil. 2. Initial characterization of the hydrolyzable fraction. J. Agric. Food Chem. 1992, 40, 1577-1580. [CrossRef] 